\title{
Hangover relieving and antioxidant effects of Gynostemma pentaphyllum (Thunb.) Makino and/or Hovenia dulcis Thunb. extracts
}

\author{
Eun Mi Park, Min Young Kim* \\ Toxicology Laboratory, Faculty of Biotechnology (Biomaterials), College of Applied Life Science, SARI, Jeju National University, Jeju, Republic of Korea.
}

\begin{tabular}{l}
\hline ARTICLE INFO \\
\hline Received on:07/04/2019 \\
Accepted on: 01/08/2019 \\
Available online: 05/10/2019
\end{tabular}

\section{Key words:}

Antioxidant, acetaldehyde dehydrogenase, aldehyde dehydrogenase, Gynostemma pentaphyllum (Thunb.)

Makino distillate. Hovenia

dulcis Thunb. extract .

\begin{abstract}
The present study attempts to study alcohol metabolizing and antioxidant properties of Gynostemma pentaphyllum (Thunb.) Makino distillate (GPD) and combination effects with Hovenia dulcis Thunb. extract (HDE) on these activities. The alcohol-metabolizing activity of GPD with/without HDE was determined by assessing alcohol dehydrogenase $(\mathrm{ADH})$ and acetaldehyde dehydrogenase (ALDH) activities. To define the effect of GPD with/without HDE on alcohol metabolism, antioxidant activities and total phenolic content of GPD with/without HD extract were evaluated using 2-diphenyl-1-picrylhydrazyl free radical scavenging, ferrous chelating assays, and the Folin-Ciocalteu method. Cytotoxicity against human normal liver CHANG cells was also evaluated using the 3-(4, 5-dimethylthiazol-2-yl)-2, 5-diphenyltetrazolium bromide assay. GPD treatment alone or in combination with HDE significantly increased ADH and ALDH activities; combined treatment was most effective. Total phenolic and flavonoid contents were greater in combination than the level found in GPD alone. GPD revealed a synergistic antioxidant effect when combined with HDE. GPD and/or HDE had no antiproliferative activity against the normal liver cell line. These results suggest that GPD-HDE combination is the possible natural resource for the management of alcohol-induced liver injury.
\end{abstract}

\section{INTRODUCTION}

Alcohol use is widespread throughout the world. Many previous works have focused on addressing alcoholism and relieving hangover because heavy alcohol drinking is associated with many social problems (Bourogaa et al., 2013). In the liver, alcohol dehydrogenase (ADH) metabolizes alcohol to acetaldehyde and then converts to acetate and water by aldehyde dehydrogenase (ALDH) (Bourogaa et al., 2013). Acetaldehyde, the metabolic intermediate of alcohol oxidation, is further oxidized into acetic acid and water by ALDH. Acetaldehyde could cause toxic effects including lightheadedness, a rapid pulse, sweating, nausea, vomiting, and functional modulation of proteins (Bourogaa et al., 2013; Lee and Park, 1999). Alcohol toxicity is mainly caused by

\footnotetext{
"Corresponding Author

Min Young Kim, Toxicology Laboratory, Faculty of Biotechnology (Biomaterials), College of Applied Life Science, SARI, Jeju National University, Jeju, Republic of Korea.E-mail: jeffmkim@jejunu.ac.kr
}

reactive oxygen species and free radicals resulting from ethanol metabolism in the liver (Lieber, 1997). Conventional or synthetic drugs for enhancing hepatic antioxidant abilities and the treatment of liver disease have been used widely (Okaiyeto et al., 2018). However, it needs to find beneficial natural hepatoprotective drugs for alcohol consumers to avoid alcoholism and alcohol-induced diseases because of side-effects of synthetic medicines.

Gynostemma pentaphyllum (Thunb.) Makino, a perennial herb distributed mostly in Korea, Japan, China, and Southeast Asia, has been reported to have a wide range of health benefits, including immunomodulatory, antitumor, nephroprotective, hepatoprotective, antimicrobial, and anti-inflammatory activities (Long, 2010; Wang et al., 2002). Furthermore, both basic and clinical studies have suggested that G. pentaphyllum (Thunb.) Makino may have modest benefits in the treatment of fatty liver disease (Hong et al., 2015). However, its effect on alcohol oxidation has yet to be reported. The present study was therefore designed to investigate the effect of G. pentaphyllum (Thunb.) Makino distillate (GPD) on hangover relief, which would be 
linked to the elimination of alcohol from the body through sweat, breath, and urine.

In traditional medicine system, mixtures of plants are used rather than one species because the mixture of the two or more species gives a better activity than either species on its own. Hovenia dulcis Thunb is a well-known natural hepatoprotective agent which has been used for the treatment of alcohol-related liver diseases and alcoholism (Hyun et al., 2010; Liang and Olsen, 2014). Even the antioxidant and alcohol relieving activity of Hovenia dulcis Thunb. has been extensively reported (Hyun et al., 2010; Liang and Olsen, 2014), there was only the action of the individual compound. In this study, the enhancing effects of Hovenia dulcis Thunb. extract (HDE) with GPD on antioxidant effect as well as ADH and ALDH activities were explored.

\section{MATERIALS AND METHODS}

\section{Plant sample preparation}

Gynostemma pentaphyllum (Thunb.) Makino used in this study was harvested in Jeju-do Province, South Korea in 2016. GPD sample was provided by Youngmul company (Jeju, Korea). Briefly, samples were washed, rinsed carefully, and then dried at room temperature for 1 week. Dried G. pentaphyllum (Thunb.) Makino $(2.8 \mathrm{~kg})$ were soaked in water $\left(\begin{array}{ll}18 & 1\end{array}\right)$ and then distilled at $116^{\circ} \mathrm{C}$ for 190 minutes using a high-speed, low-temperature vacuum extractor (DM-3000, Daehan median co., LTD, Seoul, Korea). The primary distillate was filtered to remove particles and stored at $4{ }^{\circ} \mathrm{C}$ for later use. HDE sample was prepared using a previously described protocol (Kim, 2017).

\section{ADH and ALDH activities}

Enzyme-linked immunosorbent assay kits were used to measure EtOH metabolites: ethanol and acetaldehyde. ADH and ALDH activities were measured by using an ethanol and acetaldehyde quantification assay kit (Megazyme, Wicklow, Ireland) following the manufacturer's instructions. The enzyme activity of ADH and ALDH in the control was set to $100 \%$ and the treated groups were measured in comparison to them.

\section{Antioxidant activities}

1,1-diphenyl-2-picrylhydrazyl (DPPH) free radical scavenging activity was determined as described in our recently published paper (Kim, 2017). All tests were performed in triplicate. The ferrous ion chelating ability was also determined as described earlier (Kim, 2017). Briefly, $250 \mu \mathrm{l}$ of the GPD and/or HDE was mixed with $5 \mu \mathrm{l}$ of $2 \mathrm{mM}$ ferrous chloride $\left(\mathrm{FeCl}_{2}\right)$. The reaction was initiated by the addition of $10 \mu \mathrm{l}$ of $5 \mathrm{mM}$ ferrozine and then incubated at $25^{\circ} \mathrm{C}$ for 10 minutes. The absorbance of the reaction mixtures was measured at $562 \mathrm{~nm}$ against blank samples.

\section{Total phenolic and flavonoid contents}

Total soluble phenolics were spectrophotometrically determined with Folin-Ciocalteu reagent (Sigma-Aldrich, MO) using gallic acid as the standard as reported previously (Kim, 2017). Total phenolic content was calculated as gallic acid equivalents (GAE) per liter of sample on the basis by comparison with a standard curve of gallic acid.
Total flavonoid content was determined by the spectrophotometric method of rutin based on procedures described previously (Kim, 2017). Total flavonoid content was calculated from a calibration curve of ruin and the results were expressed as the $\mathrm{mg}$ of rutin equivalents (RE) per liter of sample.

\section{In vitro cytotoxic activity}

CHANG cells were maintained in Dulbecco's modified Eagle's medium (Lonza, Walkersville, MD) supplemented with L-glutamine, antibiotics, and FBS. The in vitro cytotoxic activity was determined from the mitochondrial activity of cells which represent the number of viable cells after the treatment, by using the 3-(4,5-dimethylthiazol-2-yl)-2,5-diphenyl tetrazolium bromide (Boehringer Mannheim, Indianapolis, IN, USA) cytotoxic assay on human CHANG normal liver cells in tissue culture.

\section{Statistical analysis}

Comparisons of all results between GPD-HDE combination and GPD alone were made by using a nonparametric test (Mann-Whitney U test) with $p<0.05$ (SPSS, ver. 12.0; SPSS Inc., Chicago, IL). Three replicate measurements were performed.

\section{RESULTS AND DISCUSSION}

Alcohol metabolism is usually considered to be one of the major causes of alcohol-induced liver injury (Zakhari and $\mathrm{Li}, 2007)$. The liver is the main site of alcohol metabolism in the body. More than $85 \%-90 \%$ of ingested alcohol is metabolized in the liver (Zakhari and Li, 2007). Alcohol is metabolized to acetaldehyde by $\mathrm{ADH}$ and, subsequently, to acetic acid by ALDH. Therefore, the toxicity of alcohol is associated with the activities of ADH and ALDH (Yoo et al., 2011). Herbal hangover remedy has been commonly used for centuries. Numerous studies show that natural products have beneficial effects on alcohol metabolism in animal and human studies (Wang et al., 2016). It increased the activity of ADH and ALDH in the serum and liver along with the decreased risk of the liver (Wang et al., 2016). In this study, the ADH or ALDH activation ability was measured in GPD alone or the mixture of GPD and HDE in vitro. ADH activity was slightly increased up to $106 \%$ by GPD alone with no observed significance, whereas the mixture of GPD with HDE revealed the extremely higher ADH activity (157\%) than that of GPD alone (Table 1). However, ALDH activities were similarly increased in both individual GPD (320\%) and combination with HDE (307\%) (Table 1). Combination of GPD with HDE did not change the ALDH activity suggesting that GPD had no interaction with HDE.

Many medicinal herbs have great antioxidant potential and exhibit a wide range of biological effects such as free radical scavenging (Li et al., 2013). Previous studies described that

Table 1. ADH and ALDH activity of GPD with/without HDE.

\begin{tabular}{lcc}
\hline & ADH activity (\%) & ALDH activity (\%) \\
\hline GPD & $105.9 \pm 6.93$ & $319.7 \pm 13.01$ \\
GPD+HDE & $157.3 \pm 2.91^{*}$ & $306.9 \pm 22.95$ \\
\hline
\end{tabular}

$\mathrm{GPD}=$ G. pentaphyllum (Thunb.) Makino distillate; $\mathrm{HDE}=$ Hovenia dulcis Thunb. extract; $\mathrm{GAE}=$ Gallic acid equivalent; $\mathrm{RE}=$ Rutin equivalent. All values are expressed as mean \pm S.D $(n=3)$. "Data were statistically different from the value of GPD alone $(p<0.05)$. 
Table 2. Antioxidant activity and total phenolic and flavonoid contents of GPD with/without HDE.

\begin{tabular}{ccccc}
\hline & $\begin{array}{c}\text { DPPH free radical } \\
\text { scavenging activity } \\
(\%)\end{array}$ & $\begin{array}{c}\text { Ferrous ion } \\
\text { chelating } \\
\text { activity }(\%)\end{array}$ & $\begin{array}{c}\text { Total phenolic } \\
\text { contents } \\
(\mathbf{m g} \text { GAE/l) }\end{array}$ & $\begin{array}{c}\text { Total } \\
\text { flavonoid } \\
\text { contents } \\
\text { (mg RE/l) }\end{array}$ \\
\hline GPD & $5.5 \pm 1.74$ & $3.1 \pm 0.81$ & $16.3 \pm 0.46$ & $2.7 \pm 0.28$ \\
GPD + & $101.4 \pm 3.45^{*}$ & $89.1 \pm 1.81^{*}$ & $1,721.4 \pm 15.20^{*}$ & $1,070.6 \pm$ \\
HDE & & & & $22.94^{*}$ \\
\hline
\end{tabular}

$\mathrm{GPD}=$ G. pentaphyllum (Thunb.) Makino distillate; HDE = Hovenia dulcis Thunb. extract; GAE $=$ Gallic acid equivalent; RE $=$ Rutin equivalent. All values are expressed as mean \pm S.D $(n=3)$. "Data were statistically different from the value of GPD alone $(p<0.05)$.

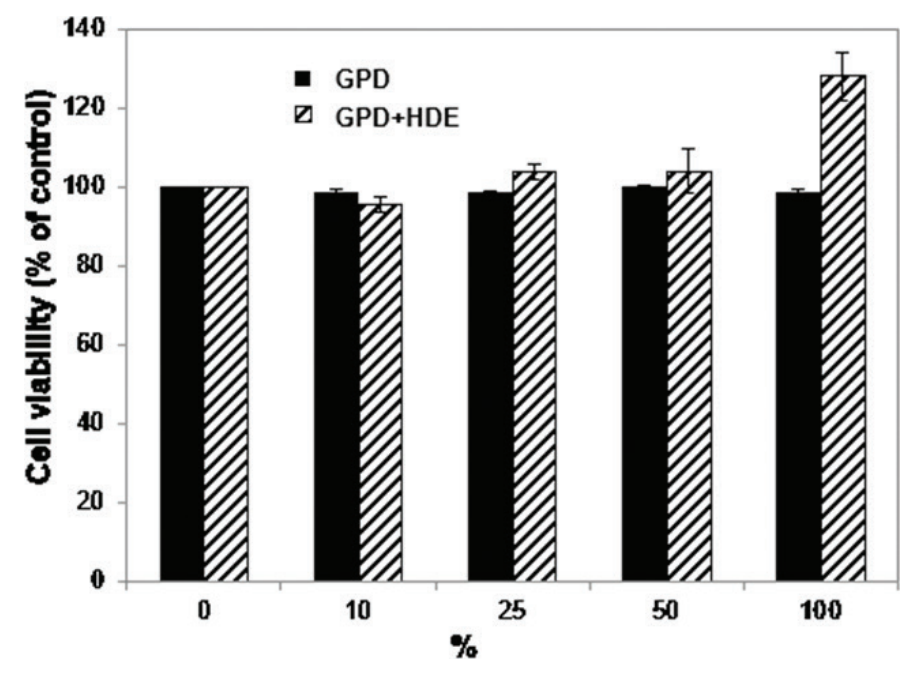

Figure 1. Cytotoxicity of GPD with/without HDE in CHANG normal liver cell lines. Values are the means \pm S.D $(n=3)$.

oxidative stress involved in alcohol metabolism causes alcoholrelated hangovers and suggests that antioxidants could alleviate hangover symptoms (Marino et al., 2004). Several natural herbs have a beneficial effect on alcohol-induced hangovers in animal and human studies (Huang et al., 2005; Simic, 1988). Due to their excellent antioxidant activity, considering the complexity of the composition of herbs, their combination is needed for prevention and treatment of alcohol-induced hangover symptoms (Huang et al., 2005). Herein, both DPPH scavenging test and the ferrous ion chelating ability were determined to evaluate the antioxidant property of the individual GPD or GPD-HDE combination. In the present study, a binary combination of GPD and HDE has demonstrated considerable strong antioxidant effects (89 and 101\%) (Table 2) indicating that concurrent usage of these plants has greatly increased the antioxidant activity, which may attenuating withdrawal syndromes of alcohol use disorder.

As is well known, flavonoids and other polyphenols lead to powerful antioxidant effects (Stevenson and Hurs, 2007). Therefore, we measured total phenolic and flavonoid contents in GPD alone or GPD-HDE combination (Table 2). Result clearly shows that the mixture of GPD and HDE (1,721 mg GAE/l and $1,071 \mathrm{mg} \mathrm{RE} / \mathrm{l}$, respectively) significantly increased $(p<0.05)$ total phenolic and flavonoid contents compared to single GPD (Table 2). We also found that the phenolic content had a positive correlation with the antioxidant capacity to scavenge the DPPH radical and chelate ferrous ion (Table 2), which is similar to previous research (Stevenson and Hurs, 2007).

The toxicological potential of a compound is analyzed through the affectation of vital functions of normal cell lines. It is important that such selection could be done at the very beginning of the developmental process, at the stage of in-vitro studies (Yahima et al., 2014). Thus, the cytotoxic effect of GPD alone or GPD-HDE combination on normal cells (human CHANG normal liver cells) was tested. We observed no antiproliferative effects of GPD alone or GPD-HDE combination on CHANG cells (Fig. 1).

The results presented in this work indicated that the GPD-HDE combination enhanced alcohol metabolizing enzyme activities and exerting antioxidant effects. Thus, GPD-HDE combination could be a new candidate therapeutics for alcoholrelated liver disease and alcohol use disorders.

\section{ACKNOWLEDGMENT}

This work was supported by the 2019 scientific promotion program funded by Jeju National University, and by Basic Science Research Program (2017R1D1A1B03028849, 2016R1A6A1A03012862 and 2014R1A1A2056292) through the National Research Foundation of Korea (NRF) funded by the Ministry of Education, Science and Technology, Republic of Korea.

\section{CONFLICTS OF INTEREST}

The authors declare that they have no conflicts of interest.

\section{REFERENCES}

Bourogaa E, Nciri R, Mezghani-Jarraya R, Racaud-Sultan C, Damak M, El Feki A. Antioxidant activity and hepatoprotective potential of Hammadascoparia against ethanol-induced liver injury in rats. J Physiol Biochem, 2013; 69(2):227-37.

Hong M, Li S, Tan HY, Wang N, Tsao SW, Feng Y. Current status of herbal medicines in chronic liver disease therapy: the biological effects, molecular targets and future prospects. Int J Mol Sci, 2015; 16(9):28705-45.

Huang D, Ou B, Prior RL. The chemistry behind antioxidant capacity assays. J Agric Food Chem, 2005; 53(6):1841-56.

Hyun TK, Eom SH, Yu CY, Roitsch T. Hovenia dulcis - an Asian traditional herb. Planta Medica, 2010; 76(10):943-9.

Kim MY. Phytochemical and antioxidant activity of Camellia mistletoe (K. japonica) extracts. Asian J Pharm Clin Res, 2017; 10(12):400-3.

Lee JH, Park KS. Effect of Ganoderma lucidum on the liver function and lipid metabolism in alcohol consuming rats. Korean J Nutr, 1999; 32(5):519-25.

Li S, Li SK, Gan RY, Song FL, Kuang L, Li HB. Antioxidant capacities and total phenolic contents of infusions from 223 medicinal plants. Ind Crops Prod, 2013; 51:289-98.

Liang J, Olsen RW. Alcohol use disorders and current pharmacological therapies: the role of GABAA receptors. Acta Pharmacol Sin, 2014; 35(8):981-93.

Lieber CS. Ethanol metabolism, cirrhosis and alcoholism. Chin J Appl Physiol, 1997; 257(1):59-84.

Long BB. The effects of gynostemma on sports ability of mice. Chin J Appl Physiol, 2010; 26:339-40.

Marino MD, Aksenov MY, Kelly SJ. Vitamin E protects against alcohol-induced cell loss and oxidative stress in the neonatal rat hippocampus. Int J Dev Neurosci, 2004; 22:363-77.

Okaiyeto K, Nwodo UU, Mabinya LV, Okoh AI. A review on some medicinal plants with hepatoprotective effects. Pharmacogn Rev, 2018; 12(24):186-99. 
Simic MG. Mechanisms of inhibition of free-radical processes in mutagenesis and carcinogenesis. Mutat Res, 1988; 202(2):377-86.

Stevenson DE, Hurst RD. Polyphenolic phytochemicals -just antioxidants or much more? Cell Mol Life Sci, 2007; 64(22):2900-16.

Wang F, Li Y, Zhang YJ, Zhou Y, Li S, Li HB. Natural products for the prevention and treatment of hangover and alcohol use disorder. Molecules, 2016; 21(1):64.

Wang QF, Chen JC, Hsieh SJ, Cheng CC, Hsu SL. Regulation of Bcl-2 family molecules and activation of caspase cascade involved in gypenosides-induced apoptosis in human hepatoma cells. Cancer Lett, 2002; 183(2):169-78.

Yahima FH, Alexis DG, Hermis RS, Jenny LR, Lianet MF, William NS. Cytotoxic effect of Cuban propolis extracts on normal cells and in vitro basal toxicity assay to estimate acute oral toxicity. Am J Essential Oils Nat Prod, 2014; 2(1):19-23.
Yoo YM, Jung EM, Kang HY, Choi IG, Choi KC, Jeung EB. The sap of Acer okamotoanum decreases serum alcohol levels after acute ethanol ingestion in rats. Int J Mol Med, 2011; 28(4): 489-95.

Zakhari S, Li TK. Determinants of alcohol use and abuse: impact of quantity and frequency patterns on liver disease. Hepatology, 2007; 46(6):2032-9.

How to cite this article:

Park EM, Kim MY. Hangover relieving and antioxidant effects of Gynostemma pentaphyllum (Thunb.) Makino and/ or Hovenia dulcis Thunb. extracts. J Appl Pharm Sci, 2019; 9(10):116-119. 